\title{
The IKK-related kinases: from innate immunity to oncogenesis
}

\author{
Jean-François Clément ${ }^{1,2}$, Sylvain Meloche ${ }^{3,4}$, Marc J Servant ${ }^{1,2}$ \\ ${ }^{1}$ Faculté de Pharmacie, Université de Montréal, C.P. 6128, succursale Centre-Ville, Montréal, Québec, Canada H3C 3J7; ${ }^{2}$ Groupe de \\ Recherche Universitaire sur le Médicament, Université de Montréal, C.P. 6128, succursale Centre-Ville, Montréal, Québec, Canada \\ H3C 3J7; ${ }^{3}$ Institut de Recherche en Immunologie et Cancérologie, Université de Montréal, C.P. 6128, succursale Centre-Ville, \\ Montréal, Québec, Canada H3C 3J7; ${ }^{4}$ Departments of Pharmacology and Molecular Biology, University of Montreal, C.P. 6128, \\ succursale Centre-Ville, Montréal, Québec, Canada H3C 3J7
}

Over the past four years, the field of the innate immune response has been highly influenced by the discovery of the IKB kinase (IKK)-related kinases, TANK Binding Kinase 1 (TBK1) and IKKi, which regulate the activity of interferon regulatory factor (IRF)-3/IRF-7 and NF-אB transcription factors. More recently, additional essential components of the signaling pathways that activate these IKK homologues have been discovered. These include the RNA helicases RIGi and MDA5, and the downstream mitochondrial effector known as CARDIF/MAVS/VISA/IPS-1. In addition to their essential functions in controlling the innate immune response, recent studies have highlighted a role of these kinases in cell proliferation and oncogenesis. The canonical IKKs are well recognized to be a bridge linking chronic inflammation to cancer. New findings now suggest that the IKK-related kinases TBK1 and IKKi also participate in signaling pathways that impact on cell transformation and tumor progression. This review will therefore summarize and discuss the role of TBK1 and IKKi in cellular transformation and oncogenesis by focusing on their regulation and substrate specificity. Keywords: cancer, IKKi, TBK1, cytokines, NF- $\mathrm{BB}$, interferon regulatory factor, inflammation Cell Research (2008) 18:889-899. doi: 10.1038/cr.2008.273; published online 12 August 2008

\section{Introduction}

The molecular links between chronic inflammation and cancer have only recently begun to emerge, despite the fact that Rudolf Virchow proposed such an association more than 100 years ago $[1,2]$. Following exposure to pathogenassociated molecular patterns (PAMPs), the innate immune response and the inflammation reaction that follows rely on evolutionarily conserved receptors termed pattern-recognition receptors (PRRs). Some of these receptors are located at the plasma membrane and include molecules such as the Toll-like receptors (TLR 1, 2, 4, 5, and 6) and scavenger receptors (CD36 and SR-A). Others occur in acidic endosomes (TLR 3, 7, 8, and 9), and still others can be found in the cytoplasm [PKR, the RIG-I-like helicases (RLHs) (RIGi and MDA5), the DNA-dependent activator of interferon (IFN) regulatory factors (IRFs) (DAI), and

Correspondence: Marc J Servant

Tel: +1-514-343-7966; Fax: +1-514-343-7073

E-mail: marc.servant@umontreal.ca the nucleotide-binding oligomerization domain (NOD) receptors] [3, 4]. Engagement of these receptors signals the activation of several transcription factors that are inactive in resting cells. One of the most important signaling cascades triggered by these receptors is the I $\mathrm{B}$ kinase (IKK) $\alpha / \beta-N F-\kappa B$ pathway leading to the induction of several key molecules involved in the clearance of incoming pathogens. Notably, several of these key molecules, such as TNF- $\alpha$, IL-6, or vascular endothelial growth factor (VEGF), are implicated in cell survival, cell proliferation and angiogenesis signaling pathways and, therefore, can influence cancer development [5-7]. Elegant gene targeting studies using mice with tissue-specific ablation of the $I K K \beta$ gene in epithelial cells, bone-marrow-derived macrophages, and hepatocytes demonstrated a complex role of the IKK $\beta-N F-\kappa B$ pathway in a variety of cancer models that are dependent on or independent of chronic inflammation $[8,9]$. Moreover, study of $I K K \alpha^{A A}$ knock-in mice, which express a catalytically inactive kinase mutant, revealed an important role for IKK $\alpha$ in metastatogenesis [10]. These important findings have recently been extensively reviewed $[2,5,11,12]$ and will not be discussed further here. This 
review focuses on recent data suggesting that additional players that control the innate immune system may be involved in cancer development.

\section{The IKK-related kinases, partners in NF-кB signal- ing}

The importance of the IKK complex in the regulation of NF- $\mathrm{KB}$ has fueled intensive research efforts by several groups leading to the discovery of the IKK-related kinases (for good reviews, see [13, 14]). Using degenerate primers containing sequences common to IKK $\alpha$ and IKK $\beta$, a portion of NF- $\kappa B$-activating kinase (NAK) was amplified by PCR [15]. This NAK, also called TANK Binding Kinase 1 (TBK1), was shown to act as an IKK-activating kinase responsible for NF- $\mathrm{NB}$ activation in response to growth factors that stimulate PKCE. TBK1 was originally identified in a yeast two-hybrid screening using TANK as the bait [16]. At almost exactly the same time, another IKK-related kinase was also characterized and named IKKe/IKKi. Peters and colleagues [17] found IKK $\varepsilon$ in a database search for proteins similar to IKK $\alpha$ and IKK $\beta$, whereas Akira's group [18] isolated IKKi in a subtractive hybridization screen of a cDNA library from lipopolysaccharide (LPS)stimulated mouse macrophages. In contrast to IKK $\alpha$, IKK $\beta$, and TBK1, which are constitutively expressed in virtually all cell types, IKKi is predominantly expressed in specific tissues such as the pancreas, thymus, spleen, and peripheral blood leukocytes [18]. It is also expressed at very low level in specific cell lines [19-21]. Importantly, IKKi mRNA can be induced in response to exposure to LPS, viral infection, or a number of NF-kB-inducing cytokines [18, 20, 22, 23]. TBK1 and IKKi display $61 \%$ sequence identity, and overexpression of both kinases induces NF- $\kappa B$ activation [16-18]. However, in contrast to IKK $\beta$, the enzymes TBK1 and IKKi phosphorylate only one (Serine 36 ) of the two phosphoacceptor sites present in I $\mathrm{B} \alpha[15,17,18]$. Why only one phosphorylation event occurs in the I $\mathrm{B} \alpha$ phosphodegron motif -DSGXXS- is unclear. Overexpression of IKKi is

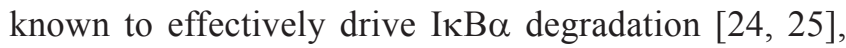
indicating that phosphorylation of Serine 36 may facilitate the phosphorylation of the protein on Serine 32, leading to its degradation. TANK phosphorylation by IKKi could also induce the release of TRAF2 followed by the subsequent activation of the IKK complex [26] (see Table 1).

Mouse embryonic fibroblasts (MEFs) deficient for IKKi

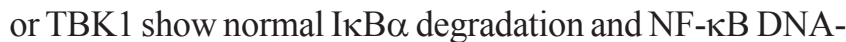
binding activity in response to classical NF- $\kappa B$ inducers including TNF $\alpha$, IL-1 $\beta$ and LPS $[22,27]$. However, the induction of well-characterized NF- $\kappa \mathrm{B}$ target genes such as $I L 6, M C P 1, C O X 2$, and ICAMI was abrogated in MEFs lacking either IKKi or TBK1. Phosphorylation of the
p65/RelA subunit of NF- $\kappa B$ was proposed to explain the observed defects in the transactivation of specific NF- $\kappa B$ target genes [27]. It is worth noting that phosphorylation of p65/RelA at Serine 536 by IKK $\beta$ and IKK $\alpha$ was shown to be an essential modification required in the second phase of NF- $\kappa \mathrm{B}$ activation. This plays a key role in determining both the strength and duration of the NF- $\mathrm{KB}$-mediated transcriptional response $[28,29]$. Interestingly, both IKKi and TBK1 also target $\mathrm{p} 65 / \mathrm{RelA}$ at Serine 536, a process clearly involved in the induction of NF- $\mathrm{KB}$-regulated genes $[30,31]$. In stimulated T cells, IKKi was shown to enhance the activity of $\mathrm{p} 65$ following its phosphorylation at Serine 468 [32]. Animal models also revealed functional similarities between IKK $\beta$ and the IKK-related kinases. Notably, TBK1-deficient mice were phenotypically similar to NEMO/IKK $\gamma$-, IKK $\beta$-, and p65-deficient mice, where embryonic lethality was observed at E14.5 due to liver degeneration and apoptosis [27]. Clearly, the IKK-related kinases are major molecular players in the modulation of inflammatory responses through their action on effectors

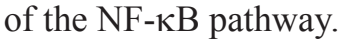

\section{The IKK-related kinases, key players in the induc- tion of type I IFN}

The field of the innate immune system was changed by the remarkable discovery that TBK1 and IKKi function as virus-activated kinases necessary for the establishment of an antiviral state [33, 34]. Both kinases phosphorylate the transcription factors IRF-3 and IRF-7 at key C-terminal residues [35-39]. IRF-3 and IRF-7 are important players in the induction of type I IFN following viral infection $[3,21,40]$. Whereas IRF-7 can be induced at the transcriptional level, IRF-3 is found latent in the cytoplasm. Upon phosphorylation of the C-terminal region by TBK1 or IKKi, IRF-3 and IRF-7 heterodimerize and translocate into the nucleus, where they induce several proinflammatory and antiviral genes such as type I IFN (see [3] for a good review). Another IRF family member, IRF-5, is also involved in type I IFN induction [41] and can also be phosphorylated by TBK1 or IKKi [42-44]. However, how IRF-5 participates in the transcriptional regulation of type I IFN genes is not yet completely understood.

Since the discovery of TBK1 and IKKi, detailed characterization of the signaling pathways involved in the activation of NF- $\mathrm{KB}$ and IRF-3 in response to pathogen infection has led to the identification of several positive scaffolding effectors regulating the phosphotransferase activities of TBK1 and IKKi. These effectors include FADD [45], TRADD [46], TRAF3 [47, 48], TANK [26, 49-51], NAP1 [30, 52], HSP90 [53], and SINTBAD [54]. In addition, intracellular RNA sensors such as RIG-I [55] 
and MDA-5 [56], which signal through the mitochondrial antiviral signaling adaptor MAVS (also known as Cardif, VISA, and IPS-1) [57-60], have been found to activate the IKK-related kinases in response to infection by RNA viruses. Cytosolic DNA sensors were also recently described. DAI, also known as DLM-1/ZBP1, is a DNA sensor that assembles with TBK1 and IRF-3 upon dsDNA treatment [61], whereas NALP3 and ASC recognize cytosolic microbial and host DNA to induce an innate immune response independent of IRFs [62]. At the level of transcriptional regulation, GRIP1 was recently identified as a new cofactor of the IRF-3 dimers [63]. Negative regulators including the SHP-2 phosphatase [64], SIKE [65], A20 [66], LGP-2 [67], NLXR1 [68], and CYLD [69] were also added to the complex puzzle. Some of these important findings have been extensively reviewed elsewhere [3, 13, 70-72] and will not be discussed further here. More recently, the IKK-related kinases have been implicated in oncogenesis $[24,25,73]$. The following discussion will describe the potential oncogenic roles of the IKK-related kinases and their different substrates (Table 1).

\section{The IKK-related kinases in oncogenesis}

In addition to the proposed roles of the IKK-related kinases in controlling NF- $\mathrm{KB}$ and the IRF transcription factors, recent findings point towards a role for TBK1 and IKKi in Ras-induced oncogenic transformation (Figure 1). TBK1 was identified as a RalB effector in the Ras-like (Ral)-guanine nucleotide exchange factor (GEF) pathway that is required for Ras-induced transformation. On the other hand, a functional screen identified IKKi as a kinase that acts downstream of the phosphatidylinositol 3-kinase (PI3K)-AKT pathway and cooperates with activated MEK to promote cellular transformation.

\section{TBK1 and RalB signaling}

The Ral GTPases RalA and RalB are two close relatives of the founding members of the Ras GTPase superfamily. The Ral proteins are involved in a variety of regulatory systems known to participate in oncogenic signaling cascades. Indeed, modulation of Ral GTPase activity influences

Table 1 Substrates of the IKK-related kinases

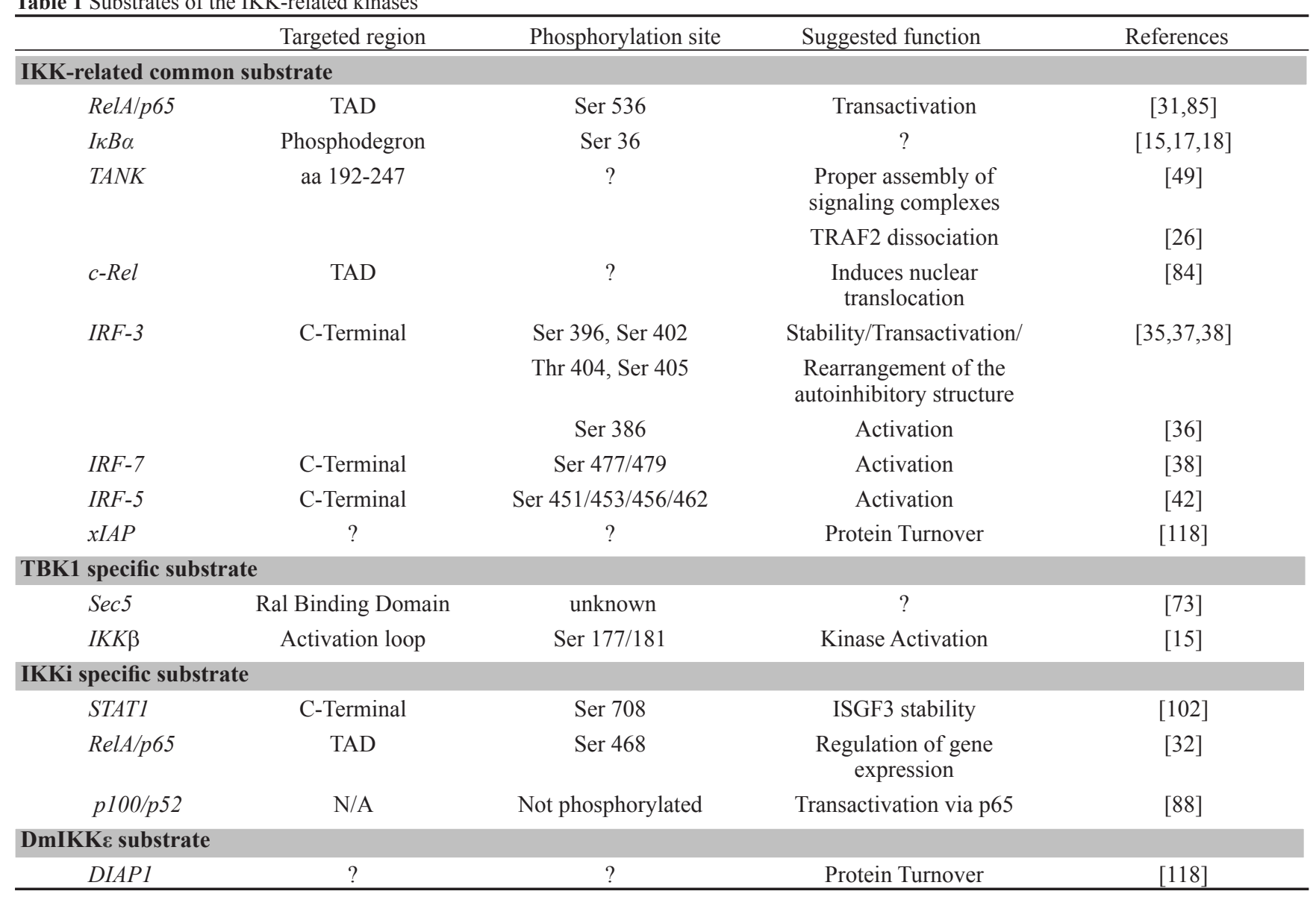


the activation of Src, JNK, and p38 kinases; induction of phospholipase D activity; and the activation of NF- $\kappa B$, Stat3, and transcription factors of the Forkhead family [74]. Moreover, Ral GEF-induced Ral activation was recently found to be necessary and sufficient for Ras transformation of a variety of human cell types [75-77]. Ral GTPases also control secretory events through the exocyst. The exocyst is a multisubunit complex whose core elements include Sec3, Sec5, Sec6, Sec8, Sec10, Sec15, Exo70, and Exo84. It is involved in the intricate secretory vesicle sorting and delivery events required to establish and support functionally and architecturally discrete plasma membrane domains [74]. Sec5 and Exo84 are both direct effectors of Ral.

While studying the contribution of Ral GTPase effector proteins to Ral function in normal and tumorigenic epithelial cells, Chien and colleagues [73] observed that RNAimediated depletion of Sec5 induces apoptosis of a variety of tumor cell lines. To understand the molecular basis of this effect, they immunoisolated the exocyst complex from epithelial cells and identified TBK1 as a co-precipitating protein. They further showed that RalB activation promotes TBK1/Sec5 assembly and that TBK1 can phosphorylate Sec5 in vitro (see Figure $2 \mathrm{~A}$ and Table 1). Silencing of TBK1 expression by RNAi was found to mimic depletion of RalB or Sec5 and induce tumor cell apoptosis. On the other hand, non-tumorigenic epithelial cells do not depend

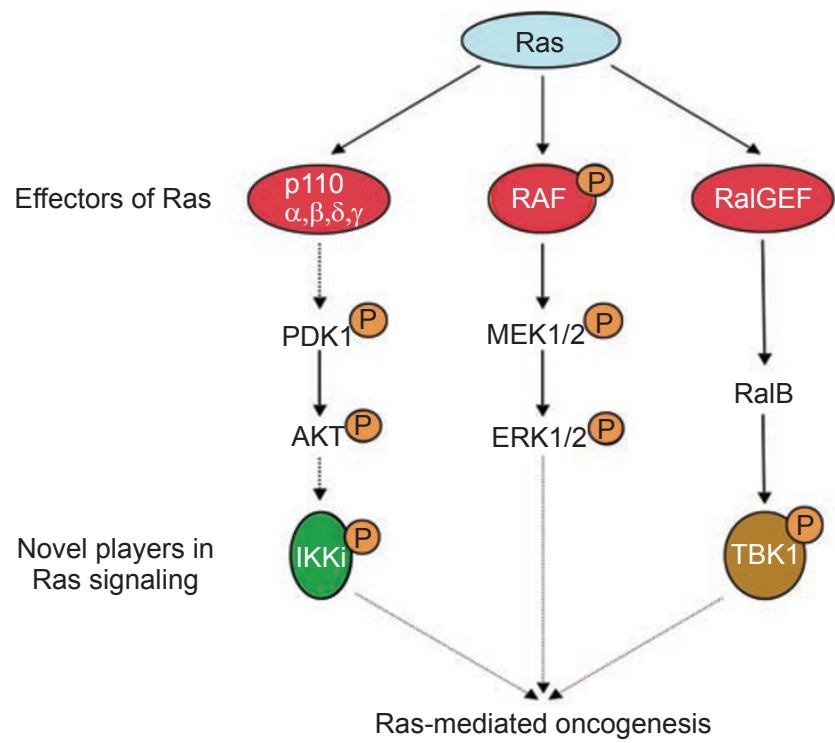

Figure 1 Pivotal contribution of the IKK-related kinases to oncogenic Ras-induced tumorigenicity. Recent findings demonstrate a role of TBK1 and IKKi in Ras-induced oncogenic transformation. In the uncontrolled proliferation of transformed cells, IKKi has a role downstream of the PI3-kinase-AKT pathway whereas TBK1 is most likely a RalB effector in the RalGEF pathway.

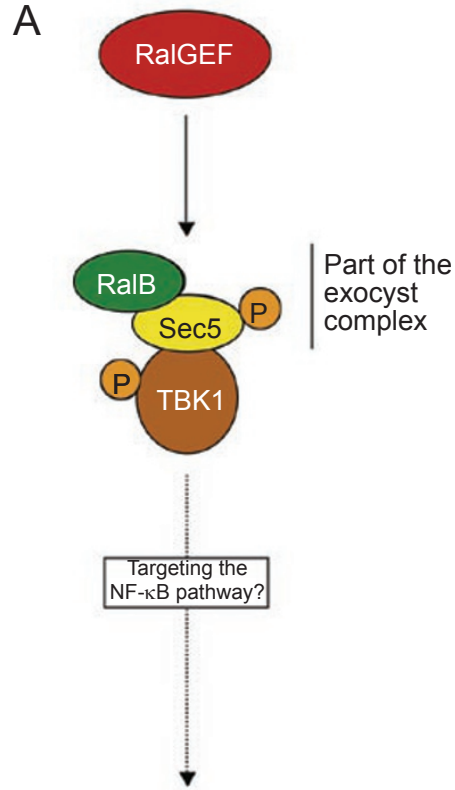

-Anchorage-independent proliferation -Survival signaling pathways

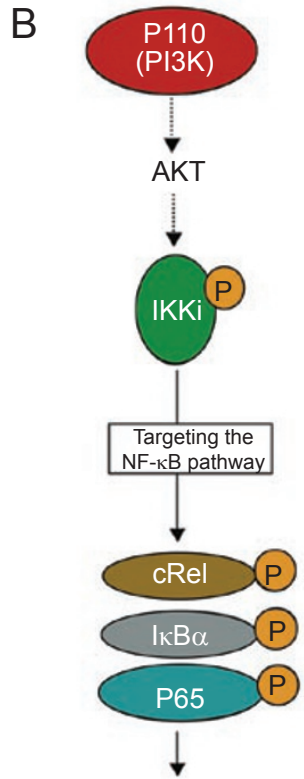

NF-kB-regulated genes: RelB; CyclinD1; MMP9; $\mathrm{BCl} 2$
Figure 2 (A) TBK1 is part of the exocyst complex and is engaged by Sec5 from within that complex. The contribution of Sec5 to TBK1 activation occurs independently of exocyst integrity. Rather, the proposed model suggests that RalB-Sec5 complex formation reveals a cryptic TBK1 interaction domain that recruits TBK1 into a kinase activation complex. The molecular mechanisms responsible for TBK1-dependent tumor cell survival remain to be characterized but could involve, in part, the NF-KB pathway. (B) IKKi is an essential effector in AKT-transformed cells. The NF- $\mathrm{B} B$ pathway is implicated in the oncogenic potential of IKKi. How the PI3-kinaseAkt pathway activates IKKi is presently unknown.

on TBK1 for survival [73]. The selective dependence of transformed cells on TBK1 was also reflected in the enzyme's phosphotransferase activity, which was higher in transformed cells than in non-tumorigenic epithelial cells. Furthermore, studies using $T B K 1^{--}$fibroblasts revealed that TBK1 expression was required for cells to tolerate transforming levels of oncogenic K-Ras ${ }^{\mathrm{G} 12 \mathrm{~V}}$. This study also established that RalB and Sec5 were required for activation of the host defense pathway. Whereas Ras activity was suppressed in non-transformed epithelial cells in response to TLR 3 signaling stimulated by poly (I:C) treatment, Ral GTPases were activated and this activation correlated with the phosphorylation of IRF-3. Moreover, siRNA-mediated depletion of Sec5 severely impaired not only the nuclear accumulation of IRF-3, but also the induction of ISG56 and $I F N \beta$ following Sendai virus $(\mathrm{SeV})$ infection or poly (I:C) treatment. In summary, the RalB/TBK1 pathway is required for the survival of Ras-transformed cells and to activate the innate immune response in non-tumorigenic 
cells (Figure 3).

Another recent study also provides evidence for a role of TBK1 in tumorigenesis. Using a genome-wide phenotypic screen based on the overexpression of 250000 cDNAs in HEK 293 cells combined with the transfer of culture supernatants onto HUVEC cells, Korherr and colleagues [78] identified TBK1 and TRIF as new regulators of vascularization. Overexpression of TRIF and TBK1 in HEK 293 cells resulted in the secretion of a complex mixture containing endothelial growth factors such as IL-8 and RANTES, as well as the anti-proliferative cytokine IFN $\beta$. The secreted mixture showed significant proangiogenic properties on endothelial cells. In addition to HEK 293 cells, the authors analyzed the proliferative response of endothelial cells to supernatants isolated from MCF-7,
PC-3, and KB-3-1 cancer cells transiently transfected with the TBK1 gene. They confirmed that the autocrine effect produced by TBK1 overexpression is observed in various cancer cell lines. Interestingly, these cancer cell lines already show increased activity of TBK1 (see above). Their study also showed some unexpected findings: TBK1 was up-regulated under hypoxic conditions, as well as in solid breast and colon tumors. This is the first report demonstrating modulation of TBK1 at the protein level.

\section{Breast cancer and the NF- $\mathrm{NB}$ pathway: is IKKi the missing link?}

Inflammatory breast cancer (IBC) is the most aggressive form of locally advanced breast cancer with a high

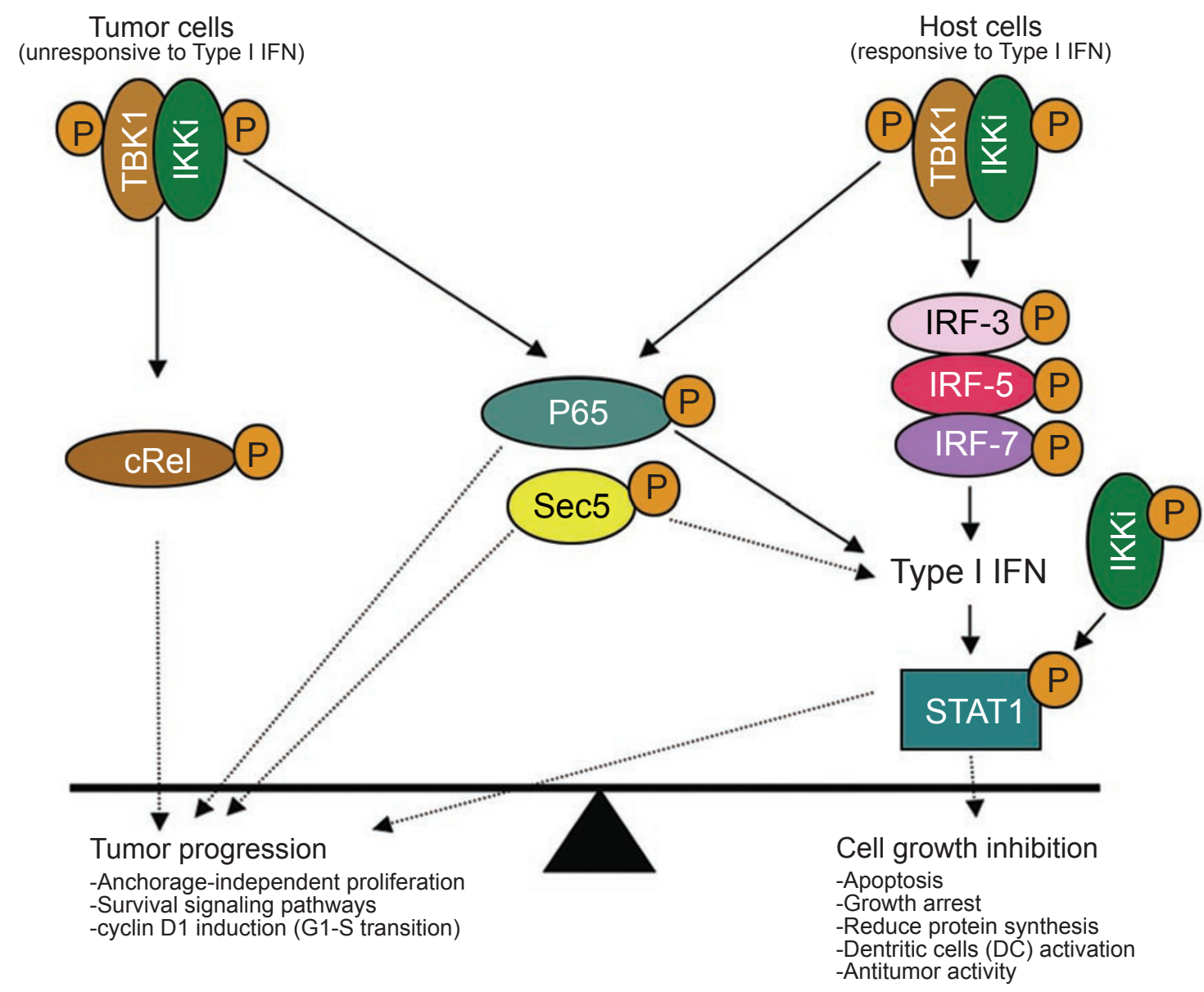

Figure 3 Transformed cells rely on the IKK-related kinases for cell proliferation and viability. Both the expression and the activity of IKKi and TBK1 can be increased in transformed tissues or cancer-derived cell lines. The IKK-related kinases depend on effectors of the NF-kB pathway, such as c-Rel and p65/RelA, in order to antagonize apoptotic signals that are normally activated when cells lose contact with the extracellular matrix or following genotoxic stimuli. Cyclin D1 induction diminishes the need of growth signal and allows G1-S transition. The role of phosphorylated Sec5 in TBK1-induced cancer cell survival remains to be elucidated. On the other hand, following exposure to PAMPs, targeted cells use the IKK-related kinases to induce a set of antiviral genes like type I IFN. IFNs are strong inducers of cell cycle arrest. However, several cancers exhibit specific mutations in components of the IFN signaling pathway and are therefore resistant to the growth-inhibitory effect of type I IFN. 
metastatic potential. At the molecular level, microarray and qPCR analysis have revealed an 'NF- $\kappa$ B signature' in IBC $[79,80]$. Interestingly, the first piece of evidence implicating IKKi in tumor development was in breast cancer. IKKi was found to be constitutively expressed in two-thirds of the human breast cancer tissues analyzed, as well as in several human breast cancer cell lines [25]. Casein kinase 2 (CK2) was proposed to be involved in the increased expression of IKKi. Most importantly, the NF- $\mathrm{kB}$ pathway is thought to contribute to the uncontrolled cellular proliferation of IKKi-expressing cells, since overexpression of an inactive mutant of IKKi reduces the expression of the cyclin D1 gene targeted by NF- $\kappa \mathrm{B}$ (Figure 2B). It also reduces the ability of breast cancer cells to grow in soft agar and to form invasive colonies in Matrigel.

Mutation of the Ras proteins or hyperactivation of downstream effector pathways, principally the mitogen-activated protein kinase (MAPK) and PI3K pathways, occurs in several epithelial cancers including breast cancers ([81] and Figure 1). An elegant functional genomic approach was used to identify protein kinases that have the potential to replace activated Akt and cooperate with a constitutively active MAPK pathway to drive tumorigenesis of immortalized human embryonic kidney epithelial cells [24]. In this study, IKKi was identified among four kinases with the transforming potential (Figure 2B). Interestingly, the authors found copy-number amplification of the 1q32 region, which includes the IKBKE locus, in $16.3 \%$ of breast cancer cell lines and in several primary human breast cancer specimens. No somatic mutation was found in the $I K B K E$ gene, and no association was observed between the $I K B K E$ copy-number gain and the estrogen receptor or Her2/neu status. IKBKE amplification correlated with overexpression of IKKi in several breast cancer cell lines such as MCF-7 and ZR-75-1. Silencing experiments with shRNAs confirmed that IKKi is required for the proliferation and survival of ZR-75-1 cells. Interestingly, knocking down the expression levels of both IKKi and TBK1 was required to diminish the viability of MCF-7 cells. This observation also supports a role for TBK1 in oncogenesis as discussed above.

At the molecular level, the overexpression of IKKi observed in breast cancer cell lines resulted in increased expression of two NF- $\mathrm{kB}$-regulated genes, MMP9 and $B C L 2$, both of which were down-regulated when IKBKE expression was suppressed. Moreover, immunohistochemical studies in patient-derived breast cancer tissue samples revealed nuclear staining for c-Rel, a recently described substrate of IKKi (Table 1). This protein had previously been reported to accumulate in the nuclei of breast cancer cells [82] and to contribute to tumorigenesis of the mammary gland in an MMTV-LTR-c-Rel transgenic mouse model [83]. IKKi modulates c-Rel activity through direct phosphorylation of the transactivation domain [84]. However, even though nuclear accumulation of c-Rel was observed in HEK 293T cells, IKKi-induced phosphorylation was insufficient to significantly induce its transactivation potential. Thus, additional signaling cascades are likely to be required for full activation of c-Rel.

In addition to c-Rel and RelB [25], RelA/p65 was observed to accumulate in the nuclei in primary human breast tumor specimens [82]. Furthermore, in many cancer cell lines, expression levels of IKKi correlate with p65 phosphorylation at Serine 536 [85]. However, Serine 536 is targeted by many other kinases, including IKK $\alpha / \beta$, TBK 1 , and RSK1 [31, 86, 87]. Finally, IKKi also interacts with $\mathrm{NF}-\kappa \mathrm{B} 2 / \mathrm{p} 100$ and p52 following TNF $\alpha$ stimulation [88]. Gel filtration experiments suggest that IKKi and p52 are components of a $600-\mathrm{kDa}$ complex. This interaction, in a ternary complex with $\mathrm{p} 65 / \mathrm{RelA}$, promotes transcription of p52-dependent genes. Although the kinase activity of IKKi is essential for this process, $\mathrm{p} 52$ does not seem to be a direct target, again suggesting that p65/RelA may be the preferred substrate of the kinase.

\section{Are other substrates of IKK-related kinases involved in tumorigenesis?}

It is intriguing that while the activity of IKK-related kinases is linked to cancer cell survival (see above), these kinases act on transcription factors with tumor suppressor properties, namely IRF-3, -5, and -7 (Table 1 and Figure $3)$. Activated by TBK1 or IKKi following viral or bacterial infections, these transcription factors ultimately lead to pathogen clearance. This occurs through the induction of type I IFN and of several IFN-stimulated genes (ISGs) involved in cellular processes of host defense. These processes include shutdown of protein translation, cell cycle withdrawal, apoptosis, and hematopoietic cell regulation such as dendritic cell (DC) activation. Notably, type I IFN signaling induces p53 accumulation [89]. Thus, at first glance, one can argue that the IKK-related kinases may also counteract tumor development through their effect on type I IFN synthesis. However, several cancers exhibit specific mutations in components of the IFN signaling pathway [90] and are therefore resistant to the growth-inhibitory effect of type I IFN. Since these cytokines are known to act by paracrine and autocrine mechanisms, transformed cells that rely on the IKK-related kinases for survival and proliferation are likely to be unresponsive to type I IFN, thus shifting the equilibrium toward tumor progression (Figure 3). On the other hand, IRF-3 is known to possess tumor suppressor activities that are unrelated to the protein's effect on type I IFN synthesis, but are directly 
linked to its effect on apoptosis. Studies have revealed that IRF-3 can transactivate a large number of genes, such as ISG56, TRAIL, and NOXA, which are known to lead to abrogation of protein synthesis or to induce apoptosis in a p53-independent manner [91-95]. IRF-3 also activates transcription of the gene encoding promyelocytic leukemia protein (PML), leading to p53-dependent growth inhibition of cancer cells $[96,97]$.

The role of IRF-7 in oncogenesis is still unclear. According to Zhang and colleagues, IRF-7 itself has oncogenic potential, as evidenced by its ability to induce tumor formation in nude mice and to cause anchorage-independent growth in NIH 3T3 cells [98]. These authors suggested that IRF-7 may cooperate with the viral oncoprotein LMP-1 in EBV-immortalized B cells or CNS lymphoma cells in order to achieve cellular transformation. Conversely, a recent study pointed out the antitumor effector functions of IRF7. Adenoviral transduction of an active form of IRF-7 into macrophages was found to up-regulate genes such as TRAIL, IL15, IL12p35, ISG56, and CD80 and also down-regulate transcription of proangiogenic/metastatic genes such as $V E G F$ and $M M P 2$. Thus, IRF-7 increases the antitumor properties of primary macrophages while reducing their protumorigenic effects [99].

IRF-5 is also a candidate tumor suppressor gene that mediates cell cycle arrest and apoptosis (reviewed in [3]). In primary hematological malignancies such as acute lymphoblastic leukemia, chronic lymphocytic leukemia, and acute myeloid leukemia, IRF-5 expression is frequently down-regulated or absent [100]. Overexpression of IRF-5 reduces colony formation of A549 lung cancer cells and HCT116 colorectal cancer cells [101], and inhibits growth of BJAB tumor cells in vivo [100].

\section{STAT1, a novel substrate of IKKi}

As key players in the IFN pathway, the signal transducers and activators of transcription (STAT) proteins contribute to the induction of numerous genes important for establishing a formidable barrier against viral infection. Recently, Tenoever and colleagues [102] established a molecular link between IKKi and the formation of the IFN-stimulated gene factor 3 complex (ISGF3), composed of the STAT1STAT2 heterodimer and IRF-9. They showed that IKKi phosphorylates STAT1 following treatment with IFN $\beta$. In vitro kinase assays combined with mass spectrometry identified Serine 708 as the phosphorylated residue. This post-translational modification allows formation of a more stable STAT1-STAT2 heterodimer, which is likely to be essential for binding the ISRE sequence located in a subset of genes involved in antiviral immunity. Thus, IKKi may be involved in defining ISGF3 binding specificity.
The STAT proteins have also been extensively studied for their role in oncogenesis. For instance, constitutive activation of STAT1, STAT3, and STAT5 has been observed in different human primary tumors and cell lines derived from breast cancer, multiple myeloma, head and neck cancer, leukemia, lymphoma, and lung cancer [103, 104]. However, unlike the clear involvement of STAT3 and STAT5 in oncogenesis, the role of STAT1 remains obscure and controversial. While STAT3 and STAT5 are clearly recognized as oncogenic transcription factors, the STAT1 pathway has been predominantly associated with the inhibition of cell proliferation and tumor suppression $[104,105]$. On one hand, STAT1 can promote tumor cell death through transcriptional regulation of genes encoding proteins such as death receptors and ligands, caspases, iNOS, Bcl-xL, and $\mathrm{p} 21^{\mathrm{Cip} 1}$ (for good reviews see [103, 106, 107]). On the other hand, according to the recent findings of Yarilina and colleagues [108], STAT1 in myeloid cells could enhance tumor progression by accelerating inflammation during the early stages of inflammation-associated tumorigenesis. Indeed, TNF $\alpha$, a well-known protumorigenic cytokine [6], can stimulate macrophages to produce low levels of IFN $\beta$, which can in turn activate JAK-STAT signaling. Once activated, JAK-STAT signaling acts in synergy with other TNF-induced signals to maintain the expression of inflammatory cytokines. It also leads to increased expression of IKKi, IRF-7, and STAT1. Thus, STAT1 activation could play opposing roles in cancer development (Figure 3 ). As mentioned above, IKKi expression is also increased in breast tumors and may therefore play a role in the constitutive activation of STAT1 seen in breast tumor-derived cell lines [103, 104].

\section{The roles of the IKK-related kinases in oncolytic- based viral therapy}

Tumor cells show deficient type I IFN signaling, a trait that can be exploited for oncolytic-based viral therapy [90]. For instance, VSV has been shown to exhibit powerful antitumor effects against metastatic breast adenocarcinoma [109-111]. The Ras-dependent MAPK pathway seems to be responsible for this down-regulation of IFN-induced antiviral response [112]. In this context, one can argue that increased levels of IKKi in breast cancer cells may promote the virus infection-mediated apoptosis. Indeed, VSV-mediated apoptosis was shown to be dependent on IRF-5, which, in turn, is likely to be phosphorylated and activated by the IKK-related kinases following VSV infection [41-44, 113]. It has also been reported that reovirus, another oncolytic virus, takes advantage of the Ras-dependent RalGEF pathway for its replication [114]. Knowing that reovirus activation of IRF-3 enhances cellular apoptosis [115], it is 
tempting to speculate that the RalB-induced TBK1 activity in transformed cells may prove useful for developing anti-cancer therapies.

\section{Conclusion}

There is now compelling evidence that the transcription factor NF- $\kappa \mathrm{B}$ plays a key role in cancer development and progression. Thus, the recent suggestion that the IKK-related kinases TBK1 and IKKi also regulate the proliferation and survival of cancer cells was not totally unexpected. However, these new findings will increase our understanding of the complex relationship between innate immune effectors and the signaling events that drive tumor formation. Some of the future questions that should be high on the priority list of investigators analyzing the role of these kinases in tumor development are the following. How is the kinase activity of TBK1 increased in cancerderived cell lines in the absence of any apparent increase in expression level? Is this related to distinct scaffold proteins that facilitate assembly of TBK1 and IKKi subcomplexes [116] or could it be more related to the decrease in the expression of the tumor suppressor CYLD [69]? What is the molecular basis of IKKi overexpression in breast tumors in the absence of amplification of the IKBKE locus, and is this related to increased NF- $\kappa B$ signaling? Are IRF-3 and IRF-7 activated in transformed cells that rely on TBK1 or IKKi for their proliferation and survival? If yes, then what is the role of the proapoptotic functions of these transcription factors that are unrelated to the proteins' effect on type I IFN synthesis? Since IKKi acts downstream of AKT in cancer-derived cell lines, is IKKi a direct substrate of AKT? Is TBK1 also subjected to this precise regulation? Are there other substrates for these kinases that could explain their effect on cellular proliferation? TBK1 and IKKi are new effectors implicated in tumor progression and may therefore represent new opportunities for drug development. Notably, the availability of compounds like SU6668, which targets TBK1 and other kinases, may help dissect the role of IKKrelated kinases in cancer [117].

\section{Acknowledgments}

We apologize to all colleagues whose papers could not be cited owing to space limitations. This work was supported by funding from the Canadian Institutes of Health Research (CIHR) to MJS (MOP-84571). MJS is the recipient of a R\&D/CIHR Health Research Foundation Career Awards in Health Sciences. SM is the recipient of a Canada Research Chair in Cellular Signaling. JFC is the recipient of a studentship from the Fonds de la Recherche en Santé du Québec (FRSQ).

\section{REFERENCES}

1 Balkwill F, Mantovani A. Inflammation and cancer: back to Virchow? Lancet 2001; 357:539-545.

2 Coussens LM, Werb Z. Inflammation and cancer. Nature 2002; 420:860-867.

3 Tamura T, Yanai H, Savitsky D, et al. The IRF family transcription factors in immunity and oncogenesis. Annu Rev Immunol 2008; 26:535-584.

4 Lee MS, Kim YJ. Signaling pathways downstream of patternrecognition receptors and their cross talk. Annu Rev Biochem 2007; 76:447-480.

5 Karin M. The IkappaB kinase - a bridge between inflammation and cancer. Cell Res 2008; 18:334-342.

6 Lin WW, Karin M. A cytokine-mediated link between innate immunity, inflammation, and cancer. J Clin Invest 2007; 117:11751183.

7 Ancrile BB, O’Hayer KM, Counter CM. Oncogenic ras-induced expression of cytokines: a new target of anti-cancer therapeutics. Mol Interv 2008; 8:22-27.

8 Maeda S, Kamata H, Luo JL, et al. IKKbeta couples hepatocyte death to cytokine-driven compensatory proliferation that promotes chemical hepatocarcinogenesis. Cell 2005; 121:977990.

9 Greten FR, Eckmann L, Greten TF, et al. IKKbeta links inflammation and tumorigenesis in a mouse model of colitis-associated cancer. Cell 2004; 118:285-296.

10 Luo JL, Tan W, Ricono JM, et al. Nuclear cytokine-activated IKKalpha controls prostate cancer metastasis by repressing Maspin. Nature 2007; 446:690-694.

11 Karin M, Greten FR. NF-kappaB: linking inflammation and immunity to cancer development and progression. Nat Rev Immunol 2005; 5:749-759.

12 Karin M, Lawrence T, Nizet V. Innate immunity gone awry: linking microbial infections to chronic inflammation and cancer. Cell 2006; 124:823-835.

13 Hacker H, Karin M. Regulation and function of IKK and IKKrelated kinases. Sci STKE 2006; 2006:re13.

14 Peters RT, Maniatis T. A new family of IKK-related kinases may function as I kappa B kinase kinases. Biochim Biophys Acta 2001; 1471:M57-62.

15 Tojima Y, Fujimoto A, Delhase M, et al. NAK is an IkappaB kinase-activating kinase. Nature 2000; 404:778-782.

16 Pomerantz JL, Baltimore D. NF-kappaB activation by a signaling complex containing TRAF2, TANK and TBK1, a novel IKKrelated kinase. EMBO J 1999; 18:6694-6704.

17 Peters RT, Liao SM, Maniatis T. IKKepsilon is part of a novel PMA-inducible IkappaB kinase complex. Mol Cell 2000; 5:513522.

18 Shimada T, Kawai T, Takeda K, et al. IKK-i, a novel lipopolysaccharide-inducible kinase that is related to IkappaB kinases. Int Immunol 1999; 11:1357-1362.

19 Gravel SP, Servant MJ. Roles of an IkappaB kinase-related pathway in human cytomegalovirus-infected vascular smooth muscle cells: a molecular link in pathogen-induced proatherosclerotic conditions. J Biol Chem 2005; 280:7477-7486.

20 Bibeau-Poirier A, Gravel SP, Clement JF, et al. Involvement of the IkappaB kinase (IKK)-related kinases tank-binding kinase $1 / \mathrm{IKKi}$ and cullin-based ubiquitin ligases in IFN regulatory fac- 
tor-3 degradation. J Immunol 2006; 177:5059-5067.

21 Honda $\mathrm{K}$, Yanai H, Negishi H, et al. IRF-7 is the master regulator of type-I interferon-dependent immune responses. Nature 2005; 434:772-777.

22 Kravchenko VV, Mathison JC, Schwamborn K, et al. IKKi/IKKepsilon plays a key role in integrating signals induced by proinflammatory stimuli. J Biol Chem 2003; 278:26612-26619.

23 Wang N, Ahmed S, Haqqi TM. Genomic structure and functional characterization of the promoter region of human IkappaB kinaserelated kinase IKKi/IKKvarepsilon gene. Gene 2005; 353:118133.

24 Boehm JS, Zhao JJ, Yao J, et al. Integrative genomic approaches identify IKBKE as a breast cancer oncogene. Cell 2007; 129:1065-1079.

25 Eddy SF, Guo S, Demicco EG, et al. Inducible IkappaB kinase/ IkappaB kinase epsilon expression is induced by CK2 and promotes aberrant nuclear factor-kappaB activation in breast cancer cells. Cancer Res 2005; 65:11375-11383.

26 Nomura F, Kawai T, Nakanishi K, et al. NF-kappaB activation through IKK-i-dependent I-TRAF/TANK phosphorylation. Genes Cells 2000; 5:191-202.

27 Bonnard M, Mirtsos C, Suzuki S, et al. Deficiency of T2K leads to apoptotic liver degeneration and impaired NF-kappaB-dependent gene transcription. EMBO J 2000; 19:4976-4985.

28 Chen LF, Williams SA, Mu Y, et al. NF-kappaB RelA phosphorylation regulates RelA acetylation. Mol Cell Biol 2005; 25:7966-7975.

29 Lawrence T, Bebien M, Liu GY, et al. IKKalpha limits macrophage NF-kappaB activation and contributes to the resolution of inflammation. Nature 2005; 434:1138-1143.

30 Fujita F, Taniguchi Y, Kato T, et al. Identification of NAP1, a regulatory subunit of IkappaB kinase-related kinases that potentiates NF-kappaB signaling. Mol Cell Biol 2003; 23:7780-7793.

31 Buss H, Dorrie A, Schmitz ML, et al. Constitutive and interleukin-1-inducible phosphorylation of p65 NF- $\{$ kappa $\}$ B at serine 536 is mediated by multiple protein kinases including I $\{$ kappa $\} \mathrm{B}$ kinase (IKK)-\{alpha\}, IKK \{beta\}, IKK \{epsilon\}, TRAF family member-associated (TANK)-binding kinase 1 (TBK1), and an unknown kinase and couples p65 to TATA-binding protein-associated factor II31-mediated interleukin-8 transcription. J Biol Chem 2004; 279:55633-55643.

32 Mattioli I, Geng H, Sebald A, et al. Inducible phosphorylation of NF-kappa B p65 at serine 468 by T cell costimulation is mediated by IKK epsilon. J Biol Chem 2006; 281:6175-6183.

33 Fitzgerald KA, McWhirter SM, Faia KL et al. IKKepsilon and TBK1 are essential components of the IRF3 signaling pathway. Nat Immunol 2003; 4:491-496.

34 Sharma S, tenOever BR, Grandvaux N, et al. Triggering the interferon antiviral response through an IKK-related pathway. Science 2003; 300:1148-1151.

35 Clement JF, Bibeau-Poirier A, Gravel SP, et al. Phosphorylation of IRF-3 on Ser 339 generates a hyperactive form of IRF-3 through regulation of dimerization and $\mathrm{CBP}$ association. $J$ Virol 2008; 82:3984-3996.

36 Mori M, Yoneyama M, Ito T, et al. Identification of Ser-386 of interferon regulatory factor 3 as critical target for inducible phosphorylation that determines activation. J Biol Chem 2004; 279:9698-9702.

37 Panne D, McWhirter SM, Maniatis T, et al. Interferon regulatory factor 3 is regulated by a dual phosphorylation-dependent switch. $J$ Biol Chem 2007; 282:22816-22822.

38 tenOever BR, Sharma S, Zou W, et al. Activation of TBK1 and IKKvarepsilon kinases by vesicular stomatitis virus infection and the role of viral ribonucleoprotein in the development of interferon antiviral immunity. J Virol 2004; 78:10636-10649.

39 Caillaud A, Hovanessian AG, Levy DE, et al. Regulatory serine residues mediate phosphorylation-dependent and phosphorylation-independent activation of interferon regulatory factor 7. $J$ Biol Chem 2005; 280:17671-17677.

40 Sato M, Suemori H, Hata N, et al. Distinct and essential roles of transcription factors IRF-3 and IRF-7 in response to viruses for IFN-alpha/beta gene induction. Immunity 2000; 13:539-548.

41 Yanai H, Chen HM, Inuzuka T, et al. Role of IFN regulatory factor 5 transcription factor in antiviral immunity and tumor suppression. Proc Natl Acad Sci USA 2007; 104:3402-3407.

42 Cheng TF, Brzostek S, Ando O, et al. Differential activation of IFN regulatory factor (IRF)-3 and IRF-5 transcription factors during viral infection. J Immunol 2006; 176:7462-7470.

43 Lin R, Yang L, Arguello M, et al. A CRM1-dependent nuclear export pathway is involved in the regulation of IRF-5 subcellular localization. J Biol Chem 2005; 280:3088-3095.

44 Schoenemeyer A, Barnes BJ, Mancl ME, et al. The interferon regulatory factor, IRF5, is a central mediator of toll-like receptor 7 signaling. J Biol Chem 2005; 280:17005-17012.

45 Balachandran S, Thomas E, Barber GN. A FADD-dependent innate immune mechanism in mammalian cells. Nature 2004; 432:401-405.

46 Michallet MC, Meylan E, Ermolaeva MA, et al. TRADD protein is an essential component of the RIG-like helicase antiviral pathway. Immunity 2008; 28:651-661.

47 Hacker H, Redecke V, Blagoev B, et al. Specificity in Toll-like receptor signalling through distinct effector functions of TRAF3 and TRAF6. Nature 2006; 439:204-207.

48 Oganesyan G, Saha SK, Guo B, et al. Critical role of TRAF3 in the Toll-like receptor-dependent and -independent antiviral response. Nature 2006; 439:208-211.

49 Gatot JS, Gioia R, Chau TL, et al. Lipopolysaccharide-mediated interferon regulatory factor activation involves TBK1-IKKepsilon-dependent Lys(63)-linked polyubiquitination and phosphorylation of TANK/I-TRAF. J Biol Chem 2007; 282:31131-31146.

50 Guo B, Cheng G. Modulation of the interferon antiviral response by the TBK1/IKKi adaptor protein TANK. J Biol Chem 2007; 282:11817-11826.

51 Rothe M, Xiong J, Shu HB, et al. I-TRAF is a novel TRAF-interacting protein that regulates TRAF-mediated signal transduction. Proc Natl Acad Sci USA 1996; 93:8241-8246.

52 Sasai M, Oshiumi H, Matsumoto M, et al. Cutting edge: NFkappaB-activating kinase-associated protein 1 participates in TLR3/Toll-IL-1 homology domain-containing adapter molecule1-mediated IFN regulatory factor 3 activation. J Immunol 2005; 174:27-30.

53 Yang K, Shi H, Qi R, et al. Hsp90 regulates activation of interferon regulatory factor 3 and TBK-1 stabilization in Sendai virus-infected cells. Mol Biol Cell 2006; 17:1461-1471.

54 Ryzhakov G, Randow F. SINTBAD, a novel component of innate antiviral immunity, shares a TBK1-binding domain with NAP1 and TANK. EMBO J 2007; 26:3180-3190.

55 Yoneyama M, Kikuchi M, Natsukawa T, et al. The RNA helicase 
RIG-I has an essential function in double-stranded RNA-induced innate antiviral responses. Nat Immunol 2004; 5:730-737.

56 Andrejeva J, Childs KS, Young DF, et al. The V proteins of paramyxoviruses bind the IFN-inducible RNA helicase, mda-5, and inhibit its activation of the IFN-beta promoter. Proc Natl Acad Sci USA 2004; 101:17264-17269.

57 Kawai T, Takahashi K, Sato S, et al. IPS-1, an adaptor triggering RIG-I- and Mda5-mediated type I interferon induction. Nat Immunol 2005; 6:981-988.

58 Meylan E, Curran J, Hofmann K, et al. Cardif is an adaptor protein in the RIG-I antiviral pathway and is targeted by hepatitis $\mathrm{C}$ virus. Nature 2005; 437:1167-1172.

59 Seth RB, Sun L, Ea CK, Chen ZJ. Identification and characterization of MAVS, a mitochondrial antiviral signaling protein that activates NF-kappaB and IRF 3. Cell 2005; 122:669-682.

$60 \mathrm{Xu} \mathrm{LG}$, Wang YY, Han KJ, et al. VISA is an adapter protein required for virus-triggered IFN-beta signaling. Mol Cell 2005; 19:727-740.

61 Takaoka A, Wang Z, Choi MK, et al. DAI (DLM-1/ZBP1) is a cytosolic DNA sensor and an activator of innate immune response. Nature 2007; 448:501-505.

62 Muruve DA, Petrilli V, Zaiss AK, et al. The inflammasome recognizes cytosolic microbial and host DNA and triggers an innate immune response. Nature 2008; 452:103-107.

63 Reily MM, Pantoja C, Hu X, et al. The GRIP1:IRF3 interaction as a target for glucocorticoid receptor-mediated immunosuppression. EMBO J 2006; 25:108-117.

64 An H, Zhao W, Hou J, et al. SHP-2 phosphatase negatively regulates the TRIF adaptor protein-dependent type I interferon and proinflammatory cytokine production. Immunity 2006; 25:919-928.

65 Huang J, Liu T, Xu LG, et al. SIKE is an IKK epsilon/TBK1-associated suppressor of TLR3- and virus-triggered IRF-3 activation pathways. EMBO J 2005; 24:4018-4028.

66 Lin R, Yang L, Nakhaei P, et al. Negative regulation of the retinoic acid-inducible gene I-induced antiviral state by the ubiquitinediting protein A20. J Biol Chem 2006; 281:2095-2103.

67 Rothenfusser S, Goutagny N, DiPerna G, et al. The RNA helicase Lgp2 inhibits TLR-independent sensing of viral replication by retinoic acid-inducible gene-I. J Immunol 2005; 175:52605268.

68 Moore CB, Bergstralh DT, Duncan JA, et al. NLRX1 is a regulator of mitochondrial antiviral immunity. Nature 2008; 451:573577.

69 Zhang M, Wu X, Lee AJ, et al. Regulation of IKK-related kinases and antiviral responses by tumor suppressor CYLD.J Biol Chem 2008; 283:18621-18626.

70 Kassel O, Herrlich P. Crosstalk between the glucocorticoid receptor and other transcription factors: molecular aspects. Mol Cell Endocrinol 2007; 275:13-29.

71 Seth RB, Sun L, Chen ZJ. Antiviral innate immunity pathways. Cell Res 2006; 16:141-147.

72 Honda K, Taniguchi T. IRFs: master regulators of signalling by Toll-like receptors and cytosolic pattern-recognition receptors. Nat Rev Immunol 2006; 6:644-658.

73 Chien Y, Kim S, Bumeister R, et al. RalB GTPase-mediated activation of the IkappaB family kinase TBK1 couples innate immune signaling to tumor cell survival. Cell 2006; 127:157170.

74 Camonis JH, White MA. Ral GTPases: corrupting the exocyst in cancer cells. Trends Cell Biol 2005; 15:327-332.

75 Hamad NM, Elconin JH, Karnoub AE, et al. Distinct requirements for Ras oncogenesis in human versus mouse cells. Genes Dev 2002; 16:2045-2057.

76 Lim KH, O Hayer K, Adam SJ, et al. Divergent roles for RalA and RalB in malignant growth of human pancreatic carcinoma cells. Curr Biol 2006; 16:2385-2394.

77 Rangarajan A, Hong SJ, Gifford A, et al. Species- and cell typespecific requirements for cellular transformation. Cancer Cell 2004; 6:171-183.

78 Korherr C, Gille H, Schafer R, et al. Identification of proangiogenic genes and pathways by high-throughput functional genomics: TBK1 and the IRF3 pathway. Proc Natl Acad Sci USA 2006; 103:4240-4245.

79 Van Laere SJ, Van der Auwera I, Van den Eynden GG, et al. Nuclear factor-kappaB signature of inflammatory breast cancer by cDNA microarray validated by quantitative real-time reverse transcription-PCR, immunohistochemistry, and nuclear factorkappaB DNA-binding. Clin Cancer Res 2006; 12:3249-3256.

80 Lerebours F, Vacher S, Andrieu C, et al. NF-kappa B genes have a major role in inflammatory breast cancer. BMC Cancer 2008; 8:41.

81 Downward J. Targeting RAS signalling pathways in cancer therapy. Nat Rev Cancer 2003; 3:11-22.

82 Sovak MA, Bellas RE, Kim DW, et al. Aberrant nuclear factorkappaB/Rel expression and the pathogenesis of breast cancer. $J$ Clin Invest 1997; 100:2952-2960.

83 Romieu-Mourez R, Kim DW, Shin SM, et al. Mouse mammary tumor virus c-rel transgenic mice develop mammary tumors. Mol Cell Biol 2003; 23:5738-5754.

84 Harris J, Oliere S, Sharma S, et al. Nuclear accumulation of cRel following C-terminal phosphorylation by TBK1/IKK epsilon. $J$ Immunol 2006; 177:2527-2535.

85 Adli M, Baldwin AS. IKK-i/IKKepsilon controls constitutive, cancer cell-associated NF-kappaB activity via regulation of Ser536 p65/RelA phosphorylation. J Biol Chem 2006; 281:2697626984.

86 Bohuslav J, Chen LF, Kwon H, et al. p53 induces NF-kappaB activation by an IkappaB kinase-independent mechanism involving phosphorylation of p65 by ribosomal S6 kinase 1. J Biol Chem 2004; 279:26115-26125.

87 Sakurai H, Chiba H, Miyoshi H, et al. IkappaB kinases phosphorylate NF-kappaB p65 subunit on serine 536 in the transactivation domain. J Biol Chem 1999; 274:30353-30356.

88 Wietek C, Cleaver CS, Ludbrook V, et al. IkappaB kinase epsilon interacts with $\mathrm{p} 52$ and promotes transactivation via $\mathrm{p} 65 . J$ Biol Chem 2006; 281:34973-34981.

89 Takaoka A, Hayakawa S, Yanai H, et al. Integration of interferonalpha/beta signalling to $\mathrm{p} 53$ responses in tumour suppression and antiviral defence. Nature 2003; 424:516-523.

90 Stojdl DF, Lichty B, Knowles S, et al. Exploiting tumor-specific defects in the interferon pathway with a previously unknown oncolytic virus. Nat Med 2000; 6:821-825.

91 Grandvaux N, Servant MJ, tenOever B, et al. Transcriptional profiling of interferon regulatory factor 3 target genes: direct involvement in the regulation of interferon-stimulated genes. $J$ Virol 2002; 76:5532-5539.

92 Kirshner JR, Karpova AY, Kops M, et al. Identification of TRAIL as an interferon regulatory factor 3 transcriptional target. $J$ Virol 2005; 79:9320-9324. 
93 Weaver BK, Ando O, Kumar KP, et al. Apoptosis is promoted by the dsRNA-activated factor (DRAF1) during viral infection independent of the action of interferon or p53. FASEB J 2001; 15:501-515.

94 Lallemand C, Blanchard B, Palmieri M, et al. Single-stranded RNA viruses inactivate the transcriptional activity of p53 but induce NOXA-dependent apoptosis via post-translational modifications of IRF-1, IRF-3 and CREB. Oncogene 2007; 26:328338.

95 Guo J, Hui DJ, Merrick WC, Sen GC. A new pathway of translational regulation mediated by eukaryotic initiation factor 3 . EMBO J 2000; 19:6891-6899.

$96 \mathrm{Kim} \mathrm{TK}$, Lee JS, Jung JE, et al. Interferon regulatory factor 3 activates p53-dependent cell growth inhibition. Cancer Lett 2006; 242:215-221.

97 Kim TK, Lee JS, Oh SY, et al. Direct transcriptional activation of promyelocytic leukemia protein by IFN regulatory factor 3 induces the p53-dependent growth inhibition of cancer cells. Cancer Res 2007; 67:11133-11140.

98 Zhang L, Zhang J, Lambert Q, et al. Interferon regulatory factor 7 is associated with Epstein-Barr virus-transformed central nervous system lymphoma and has oncogenic properties. $J$ Virol 2004; 78:12987-12995.

99 Romieu-Mourez R, Solis M, Nardin A, et al. Distinct roles for IFN regulatory factor (IRF)-3 and IRF-7 in the activation of antitumor properties of human macrophages. Cancer Res 2006; 66:10576-10585.

100Barnes BJ, Kellum MJ, Pinder KE, et al. Interferon regulatory factor 5, a novel mediator of cell cycle arrest and cell death. Cancer Res 2003; 63:6424-6431.

101 Mori T, Anazawa Y, Iiizumi M, et al. Identification of the interferon regulatory factor 5 gene (IRF-5) as a direct target for $\mathrm{p} 53$. Oncogene 2002; 21:2914-2918.

102 Tenoever BR, Ng SL, Chua MA, et al. Multiple functions of the IKK-related kinase IKKepsilon in interferon-mediated antiviral immunity. Science 2007; 315:1274-1278.

103 Bowman T, Garcia R, Turkson J, et al. STATs in oncogenesis. Oncogene 2000; 19:2474-2488.

104 Watson CJ. Stat transcription factors in mammary gland development and tumorigenesis. J Mammary Gland Biol Neoplasia 2001; 6:115-127.

105Desrivieres S, Kunz C, Barash I, et al. The biological functions of the versatile transcription factors STAT3 and STAT5 and new strategies for their targeted inhibition. J Mammary Gland Biol Neoplasia 2006; 11:75-87.
106Bromberg J, Darnell Jr JE. The role of STATs in transcriptional control and their impact on cellular function. Oncogene 2000; 19:2468-2473.

$107 \mathrm{Kim}$ HS, Lee MS. STAT1 as a key modulator of cell death. Cell Signal 2007; 19:454-465.

108 Yarilina A, Park-Min KH, Antoniv T, et al. TNF activates an IRF1-dependent autocrine loop leading to sustained expression of chemokines and STAT1-dependent type I interferon-response genes. Nat Immunol 2008; 9:378-387.

109Balachandran S, Barber GN. Vesicular stomatitis virus (VSV) therapy of tumors. IUBMB Life 2000; 50:135-138.

110 Balachandran S, Porosnicu M, Barber GN. Oncolytic activity of vesicular stomatitis virus is effective against tumors exhibiting aberrant p53, Ras, or myc function and involves the induction of apoptosis. $J$ Virol 2001; 75:3474-3479.

111 Ebert O, Harbaran S, Shinozaki K, et al. Systemic therapy of experimental breast cancer metastases by mutant vesicular stomatitis virus in immune-competent mice. Cancer Gene Ther 2005; 12:350-358.

112 Battcock SM, Collier TW, Zu D, Hirasawa K. Negative regulation of the alpha interferon-induced antiviral response by the Ras/Raf/MEK pathway. $J$ Virol 2006; 80:4422-4430.

113 Barnes BJ, Kellum MJ, Field AE, et al. Multiple regulatory domains of IRF-5 control activation, cellular localization, and induction of chemokines that mediate recruitment of T lymphocytes. Mol Cell Biol 2002; 22:5721-5740.

114 Norman KL, Hirasawa K, Yang AD, et al. Reovirus oncolysis: the Ras/RalGEF/p38 pathway dictates host cell permissiveness to reovirus infection. Proc Natl Acad Sci USA 2004; 101:1109911104.

115 Holm GH, Zurney J, Tumilasci V, et al. Retinoic acid-inducible gene-I and interferon-beta promoter stimulator-1 augment proapoptotic responses following mammalian reovirus infection via interferon regulatory factor-3. J Biol Chem 2007; 282:2195321961.

116 Chau TL, Gioia R, Gatot JS, et al. Are the IKKs and IKK-related kinases TBK1 and IKK-varepsilon similarly activated? Trends Biochem Sci 2008; 33:171-180.

117 Godl K, Gruss OJ, Eickhoff J, et al. Proteomic characterization of the angiogenesis inhibitor SU6668 reveals multiple impacts on cellular kinase signaling. Cancer Res 2005; 65:6919-6926.

118 Kuranaga E, Kanuka H, Tonoki A, et al. Drosophila IKK-related kinase regulates nonapoptotic function of caspases via degradation of IAPs. Cell 2006; 126:583-596. 